\title{
THE LONG TIME BEHAVIOR OF A FINITE AMPLITUDE SHEAR ALFVÉN WAVE IN A WARM PLASMA
}

\author{
J. A. IOnson and R. S. B. ONG \\ Department of Aerospace Engineering and Space Physics Research Laboratory, The University of \\ Michigan, Ann Arbor, MI 48105, U.S.A.
}

(Received 20 October 1975)

\begin{abstract}
The long time behavior of a monochromatic, finite amplitude shear Alfvén wave is studied by means of the Krylov-Bogoliubov-Mitropolsky perturbation technique. The plasma model is assumed to be described by the linearly non-dispersive, ideal magnetohydrodynamic equations. Non-linear coupling between the Alfvén pump wave and a free sound wave gives rise to forced magnetic sidebands. It is shown that the Alfvén pump wave as well as the magnetic sidebands steepen up in the long time scale.
\end{abstract}

\section{INTRODUCTION}

THIS paper deals with the long time evolution of a monochromatic, finite amplitude Alfvén wave propagating in a plasma parallel to a uniform, applied magnetic field. Our plasma model is described by the ideal magnetohydrodynamic equations. KAKUTANi et al. (1967) has shown that this simple model of a plasma is non-dispersive in regard to small amplitude waves, i.e. dispersion is absent in the linear (amplitude independent) dispersion relation.

In order to study the long time behavior of the waves we use the KrylovBogoliubov-Mitropolsky method of perturbation. Initially the ponderomotive force generated by the finite amplitude Aifvén pump wave drives a density osciliation non-resonantly, which couples energy in a nonlinear manner back into the pump wave. This has the effect of generating a third harmonic of the pump wave which, in a linearly non-dispersive medium, is also a normal mode. Therefore, the initial steepening of the pump wave occurs because of "feedback" from the sound wave. TANIUTI and WASHIMI (1968) develop a non-linear Schrödinger equation for this self-trapping process in a cold plasma and retain linear dispersion to lowest order. We go one step further and allow coherent interaction between a free sound wave and the Alfvén pump wave. In third-order forced magnetic sidebands are generated, and by the elimination of secular terms we derive a non-linear Schrödinger equation describing the second order Alfvén pump wave amplitude. By the elimination of secular terms in the fourth-order sound wave a similar equation is derived which governs the free sound wave to second-order. We also obtain the equation describing the amplitude of the forced magnetic sidebands since this is related to the product of the pump and sound wave amplitudes. From these equations the non-linear dispersion relation for each wave is determined. A stability criterion is then derived which incorporates the amplitude dependent non-linear correction to the linear dispersion relation. With the aid of this criterion we show that the Alfvén pump wave as well as the forced magnetic sidebands steepen up in the long time scale.

\section{FORMULATION OF THE PROBLEM}

Consider an infinite, uniform plasma in which the constant magnetic field $B_{0}$ lies along the $x$-direction. The plasma is warm with respect to the electrons but 
cold for the ions, thus allowing the existence of free ion-acoustic waves. We assume that dissipative losses are absent for both Alfvén wave and pressure fluctuations. Following KAKUTANi et al. (1967) the hydromagnetic approximation is valid provided the following criteria are satisfied:

$$
\begin{aligned}
c_{A} & \ll c, \quad \text { (non-relativistic) } \\
R_{\rho e}{ }^{-1} & \ll 1, \\
\lambda_{D} & \ll L, \\
R_{c i}{ }^{-1}+R_{c e}{ }^{-1} & \ll\left(c / c_{A}\right)^{2},
\end{aligned}
$$

where

$$
\begin{array}{rlrl}
R_{\rho e^{-}}{ }^{-1} & \equiv \omega / \omega_{\rho e} ; & \omega_{\rho e}=\frac{4 \pi n_{e} e^{2}}{m_{e}}, \\
{R_{c i}}^{-1} \equiv \omega / \omega_{c i} ; & \omega_{e i}=e B_{0} / m_{i} c, \\
R_{c e}{ }^{-1} \equiv \omega / \omega_{c e} ; & \omega_{c e}=-e B_{0} / m_{e} c, \\
c_{A} & =B_{0} /\left(4 \pi n_{0}\left(m_{e}+m_{i}\right)^{1 / 2},\right. \\
\lambda_{D}{ }^{2} & =k T / 4 \pi n_{0} e^{2}, \\
c & =\text { speed of light. }
\end{array}
$$

The satisfaction of the above conditions allows us to assume quasi-neutrality and neglect displacement currents. The two fluid equations can now be cast into a one-fluid form. KAKUTANi et al. $(1967,1968)$ and TANIUTI and WAsHimi (1968) used this description of a plasma in their studies of non-iinear hydromagnetic waves. Moreover for our purpose we shall choose a dispersionless $\left(R_{c e}, R_{c i} \rightarrow \infty\right)$ ideal magneto-hydrodynamic model with all disturbances propagating parallel to the constant external magnetic field $\mathbf{B}_{0}$. Let

$$
\begin{aligned}
\mathbf{B}_{0} & =B_{0} \hat{x}, \\
\mathbf{b} & =b \hat{y}, \\
\mathbf{v} & =v \hat{y}, \\
\mathbf{u} & =u \hat{x} .
\end{aligned}
$$

It is to be noted that for waves propagating along $\mathbf{B}_{0}$ the two possible linear polarizations are independent, so that the analysis can be applied to either polarization. We then have the following system of equations:

$$
\begin{gathered}
\frac{\partial \rho}{\partial t}+\frac{\partial}{\partial x}(\rho u)=0 \\
\rho \frac{\partial u}{\partial t}+\rho u \frac{\partial u}{\partial x}+c_{s}^{2} \frac{\partial \rho}{\partial x}+\frac{\partial}{\partial x}\left(b^{2} / 8 \pi\right)=0 \\
\rho \frac{\partial v}{\partial t}+\rho u \frac{\partial v}{\partial x}-\frac{B_{0}}{4 \pi} \frac{\partial b}{\partial x}=0 \\
\frac{\partial b}{\partial t}+\frac{\partial}{\partial x}(u b)-B_{0} \frac{\partial v}{\partial x}=0
\end{gathered}
$$


where we have used an equation of state of the form

$$
p=c_{s}^{2} \rho ; \quad c_{s}^{2}=k_{B} T_{e} / m_{i} .
$$

The approach we use in solving these equations will be that developed by KRYLOV and Bogoliubov described by Mrtropolsky (1961) and KaKuTANi and Sugmoto (1974). The essence of this perturbation method lies in the elimination of secular terms, thus allowing one to obtain a solution in the form of an asymptotic expansion. The expansion parameter $\varepsilon$ is chosen to be related to the amplitude of the finite amplitude Alfvén wave as follows:

$$
\varepsilon=0\left(|a| / B_{0}\right)
$$

where $|a|$ is defined as the amplitude of the Alfven pump wave. Along the same lines we assume that the amplitude of the free sound wave, essential in the production of forced magnetic sidebands, is related to the expansion parameter $\varepsilon$ in the following manner:

$$
\varepsilon^{2}=0\left(|c| / \rho_{0}\right)
$$

where $|c|$ is the magnitude of the second-order free sound wave and $\rho_{0}$ is the constant ambient density.

By means of the Krylov-Bogoliubov-Mitropolsky perturbation method for non-linear wave modulation we carry on the following expansion (KAKUTANI and Sugrmoto, 1974):

$$
\left(\begin{array}{l}
u \\
\rho \\
b \\
0
\end{array}\right)=\left(\begin{array}{l}
0 \\
\rho_{0} \\
0 \\
0
\end{array}\right)+\varepsilon\left(\begin{array}{l}
0 \\
0 \\
b_{1} \\
v_{1}
\end{array}\right)+\varepsilon^{2}\left(\begin{array}{l}
u_{2} \\
\rho_{2} \\
b_{2} \\
v_{2}
\end{array}\right)+\ldots
$$

We assume that each coefficient of the $\varepsilon$ power in the expansion of the magnetic field depends on $x$ and $t$ through $a, \bar{a}$, and the phase factor $\psi=k x-\omega t$. Similarly, each coefficient in the expansion of the density depends on $x$ and $t$ through $c, \bar{c}$, and $\psi$. As usual the bar indicates the complex conjugate operation. The complex amplitudes $a$ and $c$ are further assumed to be slowly varying functions of $x$ and $t$ through the relations

$$
\begin{aligned}
& \frac{\partial a}{\partial t}=\varepsilon \alpha_{1}+\varepsilon^{2} \alpha_{2}+\ldots \quad \& \quad \frac{\partial \bar{a}}{\partial t}=\varepsilon \bar{\alpha}_{1}+\varepsilon^{2} \bar{\alpha}_{2}+\ldots, \\
& \frac{\partial a}{\partial x}=\varepsilon \beta_{1}+\varepsilon^{2} \beta_{2}+\ldots \quad \& \quad \frac{\partial \bar{a}}{\partial x}=\varepsilon \bar{\beta}_{1}+\varepsilon^{2} \bar{\beta}_{2}+\ldots, \\
& \frac{\partial c}{\partial t}=\varepsilon A_{1}+\varepsilon^{2} A_{2}+\ldots \quad \& \quad \frac{\partial \bar{c}}{\partial t}=\varepsilon \bar{A}_{1}+\varepsilon^{2} \bar{A}_{2}+\ldots, \\
& \frac{\partial c}{\partial x}=\varepsilon B_{1}+\varepsilon^{2} B_{2}+\ldots \quad \& \quad \frac{\partial \bar{c}}{\partial x}=\varepsilon \bar{B}_{1}+\varepsilon^{2} \bar{B}_{2}+\ldots
\end{aligned}
$$

The coefficients $\alpha(a, \bar{a}), \beta(a, \bar{a}), A(c, \bar{c})$ and $B(c, \bar{c})$ are then adjusted to eliminate all secular terms in the perturbation expansion. It is interesting to note that had we allowed a first order sound wave, the perturbation scheme would break 
down because we then would not be able to eliminate all the secular terms. An example of such a breakdown in the case of a pure wave equation has been described by MONTGOMERY and TIDMAN (1964). The existence of a finite amplitude pump wave increases the order of the expansion parameter $\varepsilon$, which has the effect of producing a finer vernier in the time scale (i.e. a faster time scale). This faster time scale has the effect of keeping successive orders in the expansion "close", and therefore the perturbation scheme remains valid to all orders.
(a) 1 st order
$(\varepsilon)$.

The pump wave is assumed to be a finite amplitude, plane polarized, monochromatic Alfvén wave propagating parallel to an external magnetic field:

$$
\begin{gathered}
b_{1}=a \exp (i \psi)+\bar{a} \exp (-i \psi) \\
\rho=0,
\end{gathered}
$$

where $\psi=k x-\omega t$ and $\omega / k=c_{\mathrm{A}}$.

$$
\text { (b) 2nd order }\left(\varepsilon^{2}\right) \text {. }
$$

Using the first-order description we arrive at

$$
\begin{gathered}
\frac{\partial^{2} b_{2}}{\partial t^{2}}-c_{A}{ }^{2} \frac{\partial^{2} b_{2}}{\partial x^{2}}=0 \\
\frac{\partial^{2} \rho_{2}}{\partial t^{2}}-c_{s}{ }^{2} \frac{\partial^{2} \rho_{2}}{\partial x^{2}}=\frac{\partial^{2}}{\partial x^{2}}\left(b_{1}{ }^{2} / 8 \pi\right) .
\end{gathered}
$$

In obtaining equation (2.6) as a wave equation for a seculâr firee Alfvén wave we have placed the following restriction on $\left(\alpha_{1}, \beta_{1}\right)$ :

$$
\alpha_{1}+c_{A} \beta_{1}=0 \text { and } \bar{\alpha}_{1}+c_{A} \bar{\beta}_{1}=0 \text {. }
$$

The solution to equation (2.6) is chosen to be

$$
b_{2}=a_{3} \exp (i 3 \psi)+\bar{a}_{3} \exp (-i 3 \psi),
$$

where $\left(a_{3}, \bar{a}_{3}\right)$ indicate the amplitude of the third harmonic Alfven wave. The choice of a third harmonic solution results because of the appearance of a secular term in third-order. By adjusting $\left(a_{3}, \bar{a}_{3}\right)$ it is possible to eliminate this term from the analysis. Physically, the second order third harmonic represents the initial non-linear distortion (steepening) of the pump. It is generated by the coupling of the second-order forced sound wave, driven by the action of the pondermotive force of the pump, and the pump wave itself.

The solution to equation $(2.7)$ is given by

$$
\rho_{2}=\left(\frac{a^{2} k^{2} \mathrm{e}^{2 i \psi}}{2 \pi D_{s}(2 \omega, 2 k)}+\text { c.c. }\right)+\left(c \mathrm{e}^{i \psi^{\prime}}+\text { c.c. }\right),
$$

where $D_{s}(\omega, k)=-\omega^{2}+c_{s}^{2} k^{2}$,

and

$$
\psi^{\prime}=k^{\prime} x-\omega^{\prime} t ; \quad \omega^{\prime} / k^{\prime}=c_{s}
$$

The first bracket in the expression (2.10) for $\rho_{2}$ represents a forced density 
oscillation, which as shown by TANIUTI and WASHIMI (1968) may trap the Alfvén wave. A free sound wave is introduced in the second bracket in (2.10).

$$
\text { (c) } 3 \mathrm{~d} \text { order } \quad\left(\varepsilon^{3}\right) \text {. }
$$

The third-order magnetic field is described by the following equation:

$$
\begin{aligned}
\varepsilon^{3}\left(\frac{\partial^{2} b_{3}}{\partial t^{2}}-c_{A}{ }^{2} \frac{\partial^{2} b_{3}}{\partial x^{2}}\right)+\varepsilon^{2}\left(\frac{\partial^{2} b_{2}}{\partial t^{2}}-c_{A}{ }^{2} \frac{\partial^{2} b_{2}}{\partial x^{2}}\right)+\varepsilon\left(\frac{\partial^{2} b_{1}}{\partial t^{2}}-c_{A}{ }^{2} \frac{\partial^{2} b_{1}}{\partial x^{2}}\right) \\
+\varepsilon^{3}\left[\frac{B_{0}}{\rho_{0}} \frac{\partial}{\partial x} \rho_{2} \frac{\partial v_{1}}{\partial t}+B_{0} \frac{\partial}{\partial x} u_{2} \frac{\partial v_{1}}{\partial x}+\frac{\partial^{2}}{\partial x \partial t}\left(u_{2} b_{1}\right)\right]=0 .
\end{aligned}
$$

Now,

$$
\varepsilon\left(\frac{\partial^{2} b_{1}}{\partial t^{2}}-c_{\mathrm{A}}{ }^{2} \frac{\partial^{2} b_{1}}{\partial x^{2}}\right)=\varepsilon^{3}\left(2 i \omega \alpha_{2}+2 i k c_{A}^{2} \beta_{2}\right) \exp (i \psi)+c . c .
$$

and

$$
\varepsilon^{2}\left(\frac{\partial^{2} b_{2}}{\partial t^{2}}-c_{\mathrm{A}}^{2} \frac{\partial^{2} b_{2}}{\partial x^{2}}\right)=-\varepsilon^{3}\left(6 i \omega \alpha_{31}+6 i c_{\mathrm{A}}^{2} k \beta_{31}\right) \exp (i 3 \psi)+c . c .
$$

with

$$
\begin{aligned}
& \frac{\partial a_{3}}{\partial t}=\varepsilon \alpha_{31}+\varepsilon^{2} \alpha_{32}+\ldots \quad \& \quad \frac{\partial \bar{a}_{3}}{\partial t}=\varepsilon \bar{\alpha}_{31}+\varepsilon^{2} \bar{\alpha}_{32}+\ldots, \\
& \frac{\partial a_{3}}{\partial x}=\varepsilon \beta_{31}+\varepsilon^{2} \beta_{32}+\ldots \quad \& \quad \frac{\partial \bar{\beta}_{3}}{\partial t}=\varepsilon \bar{\beta}_{31}+\varepsilon^{2} \bar{\beta}_{32}+\ldots .
\end{aligned}
$$

The functions $\left(\alpha_{2}, \beta_{2}\right)$ can be adjusted to eliminate the first harmonic secular terms generated by the interaction of $\rho_{2}$ and $b_{1}$, i.e.

$$
i\left(\alpha_{2}+c_{A} \beta_{2}\right)=\frac{c_{A} k^{3}|a|^{2} a}{4 \pi \rho_{0} D_{s}(2 \omega, 2 k)},
$$

where $D_{s}(2 \omega, 2 k)=-4 \omega^{2}+4 c_{s}^{2} k^{2} \neq 0$

and

$$
\begin{array}{ll}
\alpha_{2}=\frac{\partial a}{\partial t_{2}} ; & t_{2}=\varepsilon^{2} t \\
\beta_{2}=\frac{\partial a}{\partial x_{2}} ; & x^{2}=\varepsilon^{2} x .
\end{array}
$$

In the same way $\left(\alpha_{31}, \beta_{31}\right)$ are adjusted to eliminate the third harmonic secular terms resulting from the coupling of $\rho_{2}$ and $b_{1}$, i.e.

$$
i\left(\alpha_{31}+c_{A} \beta_{31}\right)=\frac{3 c_{A} k^{3} a^{3}}{4 \pi \rho_{0} D_{s}(2 \omega, 2 k)} .
$$

With the aid of equation (2.5), (2.9) and (2.10) the solution to equation (2.11) can now be written as:

$$
\begin{aligned}
b_{3}=\left[a c b_{+} \exp \left(i \psi_{+}\right) / D_{A}\left(\omega_{+}, k_{+}\right)\right] \\
+\left[a \bar{c} b_{-} \exp \left(i \psi_{-}\right) / D_{A}\left(\omega_{-}, k_{-}\right)\right]+a_{4} \exp (i 4 \psi)+c . c .
\end{aligned}
$$


where

and

$$
\begin{aligned}
& b_{+}=c_{A}\left(\omega k_{+}-k c_{s} k_{+}-\beta^{1 / 2} k_{+} \omega_{+}\right) / \rho_{0} \\
& b_{-}=c_{A}\left(\omega k_{-}-k c_{s} k_{-}-\beta^{1 / 2} k_{-} \omega_{-}\right) / \rho_{0},
\end{aligned}
$$

$$
\begin{aligned}
\beta & \equiv c_{s}{ }^{2} / c_{A}{ }^{2} \\
D_{A}\left(\omega_{ \pm}, k_{ \pm}\right) & =-\omega_{ \pm}{ }^{2}+c_{A}{ }^{2} k_{ \pm}{ }^{2} \neq 0, \\
\omega_{ \pm} & =\omega \pm \omega^{\prime} \\
k_{ \pm} & =k \pm k^{\prime} .
\end{aligned}
$$

The first two terms in the expression (2.14) for $b_{3}$ represent the forced magnetic sidebands. It is important to note that the sidebands (as written above) are not normal mode Alfvén waves. The $\psi_{+}$sideband represents the "beating" of the pump with the sound wave:

$$
\begin{array}{ll}
(\omega, k) \text { mans } & \omega \rightarrow \omega_{+}-\omega^{\prime} \\
\left(\omega^{\prime}, k^{\prime}\right) \text {. } & k \rightarrow k_{+}-k^{\prime} .
\end{array}
$$

It would never satisfy the rule for a "decay" instability as this requires $\psi_{+}$to be a normal mode Alfvén wave.

The $\psi_{-}$sideband is slightly different from the $\psi_{+}$wave. In this situation we are dealing with the scattering of the pump wave off an ion-acoustic wave:

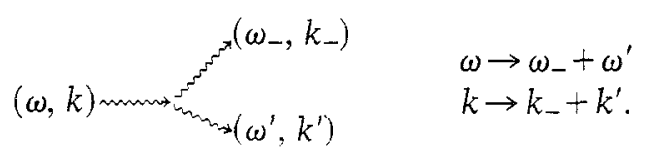

In this case the conservation relations for a decay instability are satisfied provided $\psi_{-}$is a normal mode Alfvén wave. It is a simple matter to determine $\left(\omega^{\prime}, k^{\prime}\right)$ for which the decay instability occurs. These values are

$$
\omega^{\prime}=2 \omega /\left[1+\left(c_{A} / c_{s}\right)\right] ; \quad k^{\prime}=2 k /\left[1+\left(c_{S} / c_{A}\right)\right] .
$$

The linear properties of this instability are discussed by LASHMORE-DAVrES and ONG (1974). When the conditions for the decay instability are satisfied, the last

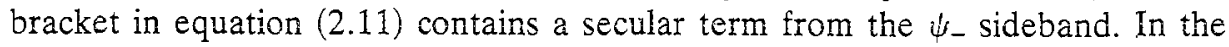
spirit of the Krylov-Bogoliubov-Mitropolsky perturbation technique this term would have to be eliminated by adding a 1st harmonic term into the solution for $b_{2}$ and adjusting its amplitude accordingly. A long time analysis of the "decay" products then simply prolong the study of the forced, scattered magnetic wave (not a normal mode) to higher order. For this reason we disregard the decay instability and proceed to study the long time behavior of the forced magnetic sidebands by restricting ourselves to the case where $D_{A}\left(\omega_{-}, k_{-}\right) \neq 0$.

The third-order sound wave is described by the following equation:

$$
\begin{array}{r}
\frac{\partial^{2} \rho_{3}}{\partial t^{2}}-c_{s}{ }^{2} \frac{\partial^{2} \rho_{3}}{\partial x^{2}}=i \frac{a k}{\pi}\left[\frac{4 k^{2} c_{A}{ }^{2}}{D_{s}(2 \omega, 2 k)}(\beta-1)+1\right] \beta_{1} \exp (i 2 \psi) \\
\quad-\left(a_{3} \bar{a} k^{2} / 4 \pi\right) \exp (i \psi)-9 a a_{3} k^{2} \exp (i 3 \psi) / 4 \pi+\text { c.c. }
\end{array}
$$

with the first order secularity condition

$$
A_{1}+c_{s} B_{1}=0 \quad \& \quad \bar{A}_{1}+c_{s} \bar{B}_{1}=0 .
$$


The solution of equation (2.16) is

$$
\begin{aligned}
\rho_{3}=-a_{3} \bar{a} k^{2} \exp (i \psi) / 4 \pi D_{s}(\omega, k) \\
+i a k\left\{\left[4 k^{2} c_{A}^{2}(\beta-1) / D_{s}(2 \omega, 2 k)\right]+1\right\} \beta_{1} \exp (i 2 \psi) / 4 \pi D_{s}(2 \omega, 2 k) \\
\quad-9 a_{3} a k^{2} \exp (i 3 \psi) / 4 \pi D_{s}(3 \omega, 3 k)+c_{2} \exp \left(i 2 \psi^{\prime}\right)+c . c .
\end{aligned}
$$

Since the ideal MHD equations completely neglect the displacement current and charge separation, the linear dispersion normally found for an ion-acoustic wave is absent. Consequently, to this order, the sound wave steepens via the generation of harmonics which are all normal modes of the plasma. The last term in (2.17) eliminates the secular second harmonic found in the fourth order.

$$
\text { (d) } 4 \text { th order }\left(\varepsilon^{4}\right)
$$

The fourth order sound wave is described by the following equation:

$$
\begin{aligned}
\varepsilon^{4}\left(\frac{\partial^{2} \rho_{4}}{\partial t^{2}}-c_{s}{ }^{2} \frac{\partial^{2} \rho_{4}}{\partial x^{2}}\right)+\varepsilon^{2}\left[\frac{\partial^{2} \rho_{2}}{\partial t^{2}}-c_{s}{ }^{2} \frac{\partial^{2} \rho_{2}}{\partial x^{2}}-\frac{\partial^{2}}{\partial x^{2}}\left(b_{1}{ }^{2} / 8 \pi\right)\right] \\
+\varepsilon^{3}\left[\frac{\partial^{2} \rho_{3}}{\partial t^{2}}-c_{s}{ }^{2} \frac{\partial^{2} \rho_{3}}{\partial x^{2}}-\frac{\partial^{2}}{\partial x^{2}}\left(b_{1} b_{2} / 4 \pi\right)\right] \\
+\varepsilon^{4}\left[\frac{\partial^{2}}{\partial x \partial t} \rho_{2} u_{2}-\rho_{0} \frac{\partial}{\partial x} u_{2} \frac{\partial u_{2}}{\partial x}-\frac{\partial^{2}}{\partial x^{2}}\left(b_{2}{ }^{2} / 8 \pi\right)-\frac{\partial^{2}}{\partial x^{2}}\left(b_{1} b_{3} / 4 \pi\right)\right]=0 .
\end{aligned}
$$

The second harmonic secular term can be eliminated by adjusting $\left(c_{2}, \bar{c}_{2}\right)$ accordingly. However, we are primarily concenned with the amplitude of the first harmonic sound wave and this is described by the first harmonic secular terms. Therefore, we have

$$
i\left(A_{2}+c_{s} B_{2}\right)=|a|^{2} c k^{\prime}\left\{\left[b_{+} / D_{A}\left(\omega_{+}, k_{+}\right)\right]+\left[b_{-} / D_{A}\left(\omega_{-}, k_{-}\right)\right]\right\} / 8 \pi c_{s},
$$

where

$$
\begin{aligned}
A_{2} & =\frac{\partial c}{\partial t_{2}}, & B & =\frac{\partial c}{\partial x_{2}} \\
t_{2} & =\varepsilon^{2} t, & x_{2} & =\varepsilon^{2} x .
\end{aligned}
$$

In summary we are then left with the following coupled equations for the sound wave and the Alfvén pump wave amplitudes:

$$
\begin{aligned}
i\left(\frac{\partial c}{\partial t_{2}}+c_{s} \frac{\partial c}{\partial x_{2}}\right)= & |a|^{2} c k^{\prime}\left\{\left[b_{+} / D_{A}\left(\omega_{+}, k_{+}\right)\right]+\left[b_{-} / D_{A}\left(\omega_{-}, k_{-}\right)\right]\right\} / 8 \pi c_{s}, \\
& i \frac{\partial a}{\partial t_{2}}+c_{A} \frac{\partial a}{\partial x_{2}}=-k c_{A}|a|^{2} a / 4 B_{0}{ }^{2}
\end{aligned}
$$

along with their complex conjugates.

\section{DERIVATION OF THE DISPERSION RELATIONS}

Without formally solving the two equations (2.20) and (2.21) a relatively large amount of qualitative information can be inferred by determining the non-linear dispersion relations for the various waves. Since linear dispersion is absent in all 
cases, knowledge of the non-linear dispersion is necessary in determining the stability of these waves.

We assume that the amplitudes $(a, \bar{a})$ of the Alfvén pump wave and $(c, \bar{c})$ of the sound wave are slowly varying, and are given by

where

$$
\mathrm{a}=\mathrm{A}_{0} \exp \left[i\left(K \xi-\Omega t_{2}\right)\right]
$$

where

$$
\begin{aligned}
\xi & =x_{1}-c_{A} t_{1} \\
t_{2} & =\varepsilon^{2} t=\varepsilon t_{1} \\
c & =C_{0} \exp \left[i\left(K^{\prime} \xi^{\prime}-\Omega^{\prime} t_{2}\right)\right],
\end{aligned}
$$

$$
\begin{aligned}
& \xi^{\prime}=x_{1}-c_{s} t_{1} \\
& t_{2}=\varepsilon^{2} t=\varepsilon t_{1} .
\end{aligned}
$$

Using equation (3.1) in (2.21) we obtain

$$
\Omega=-\left|A_{0}\right|^{2} k c_{A} / 4 B_{0}^{2} \text {. }
$$

Recalling that

$$
b_{1}=a \exp [i(k x-\omega t)]+c . c .
$$

we have

$$
b_{1}=A_{0} \exp \left[i(k+\varepsilon K) x-i\left(\omega+\varepsilon K c_{A}+\varepsilon^{2} \Omega\right) t\right],
$$

where we let

$$
\omega\left(\tilde{k}+\dot{\Delta} \tilde{k}, \dot{A}_{0}\right)=\left(\omega_{0}+c_{A} \dot{\Delta} \dot{k}\right)-\frac{\varepsilon^{2} k c_{A}\left|A_{0}\right|^{2}}{4 B_{0}^{2}}
$$

with $\Delta k=\varepsilon K$ and $\omega_{0}=k c_{A}$.

The first bracket in (3.3) is the term obtained by carrying out a Taylor series expansion about $k$, and it is due to linear dispersion. Since our ideal magnctohydrodynamic model lacks linear dispersion, the change in wave number $\Delta k$ is zero. Hence we are left with

$$
\omega\left(k, A_{0}\right)=k c_{A}\left[1-(1 / 4)\left|A_{0}\right|^{4} / B_{0}^{4}\right],
$$

where we have used $\varepsilon^{2}=\left|A_{0}\right|^{2} / B_{0}{ }^{2}$ for the Alfvén pump wave as mentioned previously in Section 2. To this order the non-linear phase and group velocities are given by

$$
c_{A}{ }^{N L}=c_{A}\left[1-(1 / 4)\left|A_{0}\right|^{4} / B_{0}^{4}\right] .
$$

Note that even to this order the phase and group velocities are equal in the non-linear limit.

Proceeding in the same way, and assuming a low $\beta \equiv c_{s}^{2} / c_{A}{ }^{2}$ plasma, the non-linear dispersion relation for the forced sidebands can be written as follows:

$$
\begin{aligned}
& \omega_{+}=\left(\omega+\omega^{\prime}\right)+\alpha_{+} A_{+}{ }^{2} \\
& \omega_{-}=\left(\omega-\omega^{\prime}\right)+\alpha_{-} A_{-}{ }^{2},
\end{aligned}
$$

where

$$
A_{+}=A_{-}=A_{0} C_{0}
$$


and

$$
\begin{aligned}
& \alpha_{+}=\varepsilon^{2} c_{\mathrm{A}} k^{\prime} / B_{0}^{2} \sqrt{\beta}\left(4-\frac{k^{\prime 2}}{k^{2}}\right) C_{0}^{2} \\
& \alpha_{-}=-\alpha_{+}
\end{aligned}
$$

$A_{0}, A_{+}, A_{-}$and $C_{0}$ represent the amplitudes of the Alfven pump wave, the $\psi_{+}$ and $\psi_{-}$magnetic sidebands, and the sound wave respectively.

We take the case where $\left(k, k^{\prime}\right)$ are greater than zero and note that from linear theory the sidebands are unstable only for $k^{\prime}>2 k$ (Lashmore-Davies). The analysis leading to equation (3.6) is not valid for the resonance case $k^{\prime}=2 k$. This singular case corresponds to the well-known decay instability where some of the excited waves are in resonance with a natural mode of the plasma. This situation has been briefly discussed in the paragraph leading to equation (2.15) in Section 2 .

\section{STABILITY ANALYSIS}

The evolution of a finite amplitude monochromatic wave in a weakly nonlinear dispersion medium proceeds via the propagation of local values for the wave number and amplitude at the non-linear (amplitude dependent) group velocity. In general we can write the non-linear dispersion relation in the form

$$
\omega(k, a)=\omega_{0}(k)+\alpha(k) a^{2},
$$

where $\omega_{0}(k)$ corresponds to the frequency of the linear wave, and the second term to the non-linear correction. The wave amplitude is denoted by a. Following KADOMTSEV and KARPMAN (1971) we now derive a stability criterion retaining the dependence of the non-linear correction term in the dispersion relation (4.1).

Let the phase of our initially monochromatic wave be

$$
\psi=k x-\omega t
$$

Then the local frequency and wave number can be expressed by

$$
k=\partial \phi / \partial x \text { and } \omega=-\partial \psi / \partial t \text {. }
$$

As a consequence we have the relations

$$
\frac{\partial k}{\partial t}=-\frac{\partial \omega}{\partial x}
$$

Using the dispersion relation (4.1) in relation (4.2) we get

where

$$
\frac{\partial k}{\partial t}+V_{g}(k, a) \frac{\partial k}{\partial x}+\alpha \frac{\partial a^{2}}{\partial x}=0
$$

$$
V_{\mathrm{g}}(k, a)=\frac{\mathrm{d} \omega_{0}(k)}{\mathrm{d} k}+a^{2} \frac{\mathrm{d} \alpha}{\mathrm{d} k}=V_{80}+a^{2} \frac{\mathrm{d} \alpha}{\mathrm{d} k},
$$

and $V_{g 0}$ is the linear group velocity. From the conservation of energy we have

$$
\frac{\partial a^{2}}{\partial t}+\frac{\partial}{\partial x}\left[a^{2} V_{g}(k, a)\right]=0 \text {. }
$$


Let us suppose we subject the monochromatic wave to a small perturbation of the form:

$$
\begin{aligned}
& k=k_{0}+k^{\prime} \exp [i(\kappa x-\nu t)] \\
& a=a_{0}+a^{\prime} \exp [i(\kappa x-\nu t)] .
\end{aligned}
$$

Substituting this into equations (4.3) and (4.4), keeping only terms linear in the perturbation yields two homogeneous algebraic equations for $k^{\prime}$ and $a^{\prime}$. We then set the coefficient determinant equal to zero and obtain a quadratic equation for $\nu$. The solution for $\nu$ is given by

$$
\begin{aligned}
\nu=\kappa V_{\mathrm{g}}-\frac{1}{2} a_{0}{ }^{2} \kappa \frac{\mathrm{d} \alpha}{\mathrm{d} k} & \\
& \pm a_{0} \kappa\left(-\frac{7}{4} a_{0}{ }^{2} \frac{\mathrm{d}^{2} \alpha}{\mathrm{d} k^{2}}-2 V_{\mathrm{g} 0} \frac{\mathrm{d} \alpha}{\mathrm{d} k}+\alpha \frac{\mathrm{d} V_{g 0}}{\mathrm{~d} k}+\alpha a_{0}{ }^{2} \frac{\mathrm{d}^{2} \alpha}{\mathrm{d} k^{2}}\right)^{1 / 2} .
\end{aligned}
$$

As described by KADOMTSEV and KARPMAN (1971) this is an instability of the type of breakdown of the wave into packets and subsequent self-contraction of the wave packets, eventually developing into "envelope solitons". On the other hand a "stable" wave would steepen in a hyperbolic sense, i.e. the system possesses real characteristics and unless additional dispersive effects come into play the wave train continues to steepen and eventually break.

In our case the Alfvén pump wave and magnetic sidebands are linearly non-dispersive, i.e. the derivative of the linear group velocity $V_{g 0}$ with respect to the wave number vanishes. Applying the stability criterion (4.5) to equations (3.4) and (3.5) with $k^{\prime}>2 k$ we find that for the Alfvén pump wave as well as for the two magnetic sidebands the left-hand side of (4.5) is real. Thus in the sense of Kadomtsev and Karpman these waves are "stable", i.e. they steepen in a typically hyperbolic fashion.

\section{CONCLUSION}

By means of an ideal magnetohydrodynamic model we have studied the long time behavior of a monochromatic, plane polarized finite amplitude Alfvén wave propagating parallel to a uniform applied magnetic field. The use of the KrylovBogoliubov-Mitropolsky perturbation method shows the development of forced magnetic sidebands, and yields two non-linear Schrödinger equations coupling the amplitudes of the Alfvén pump wave and a free sound wave. The amplitudes of the sidebands are found to be proportional to the product of the Alfvén pump and sound wave amplitudes. The non-linear dispersion relations for these waves are determined. As frequency dispersion is absent in the linear case because of the simplicity of the ideal MHD model employed, we derive a stability criterion which incorporates the amplitude dependent non-linear correction to the linear dispersion relation. Applying this to our analysis we show that the Alfvén pump wave and the forced magnetic sidebands steepen in a typically hyperbolic fashion. Therefore it is possible that the Alfvén pump wave as well as the sidebands form shocks and this phenomenon could explain the observed discontinuities in Alfvén fluctuations found by BeLCHER and DAvis (1971). 
The most serious oversight in the above analysis is the neglect of linear dispersion inherent in the ideal MHD fluid model adopted. As discussed in Section 2 this is a consequence of taking the limits

$$
\begin{aligned}
& R_{c i}=\omega_{c i} / \omega \rightarrow \infty \\
& R_{c e}=\omega_{c e} / \omega \rightarrow \infty .
\end{aligned}
$$

In higher order the linear dispersion may well be comparable to the amplitude dependent non-linear dispersion and should be taken into account.

Acknowledgments-This work is partially supported by Grant AFOSR-76-2904 and by NSF Grant ATM73-00322 A01. One of the authors (R. S. B. Ong) acknowledges useful discussions with Dr. C. N. Lashmore-Davies when he was visiting the UKAEA Culham Laboratory. The hospitality of the Theory Division of the Culham Laboratory is also greatly appreciated.

\section{REFERENCES}

Belcher J. W. and Davis L. (1971) J. geophys. Res. 76, 3534.

Bogoziubov N. N. and Mitropolsky Y. A. (1961) Asymptotic Methods in the Theory of Non-Linear Oscillations, Chapter 1. Hindustan Pub. Co., Delhi.

Kadomtsev B. B. and KarpMan V. I. (1971) Soviet Phys. Usp. 14, 40.

KakUtani T., Kawahara T. and TANiUti T. (1967) J. phys. Soc. Japan 23, 1138.

Kakutani T., Ono H., TANiuti T. and Wei C. C. (1968) J. phys. Soc. Japan 24, 1159.

Kakltani T. and Slgmoto N. (1974) Physics Fluids 17, 1617.

Lashmore-Davies C. N. (1976) Physics Fluids 19, 587.

Lashmore-Davies C. N. and ONG R. S. B. (1974) Phys. Rev. Lett 32, 1172.

Montcomery D. and Tidman D. (1964) Physics Fluids 7, 242.

TAnilti T. and Washimi H. (1968) Phys. Rev. Lett 21, 209. 